\title{
Overcoming hypoxia-induced chemoresistance to cisplatin through tumor oxygenation monitored by optical imaging
}

Donghui Song1, ${ }^{1 *}$, André O'Reilly Beringhs ${ }^{1, *}$, Zhenwu Zhuang2, Gaurav Joshi1 ${ }^{1, \#,}$, Thanh Huyen Tran1,\$, Kevin P. Claffey $^{3}$, Hong Yuan ${ }^{4}$, Xiuling $\mathrm{Lu}^{1 凶}$

1. Department of Pharmaceutical Sciences, University of Connecticut, Storrs, Connecticut, USA 06269

2. Section of Cardiovascular Medicine, Department of Medicine, Yale University School of Medicine, New Haven, CT, USA 06519

3. Department of Cell Biology, University of Connecticut Health Center, Farmington, Connecticut, USA 06030

4. Department of Radiology, School of Medicine, University of North Carolina at Chapel Hill, North Carolina, USA 27599

${ }^{*}$ Equal contributors to this work.

\#Current Address: Harvard T.H. Chan School of Public Health, Boston, Massachusetts, USA 02115

\$Current Address: Eli Lilly and Company Global Headquarters Lilly Corporate Center, Indianapolis, Indiana, USA 46285

$\triangle$ Corresponding author: Xiuling Lu, xiuling.lu@uconn.edu. University of Connecticut - School of Pharmacy, 69 N Eagleville Rd Unit 3092 , Storrs, CT USA 06269-3092.

(C) Ivyspring International Publisher. This is an open access article distributed under the terms of the Creative Commons Attribution (CC BY-NC) license (https://creativecommons.org/licenses/by-nc/4.0/). See http://ivyspring.com/terms for full terms and conditions.

Received: 2019.04.30; Accepted: 2019.05.18; Published: 2019.05.22

\begin{abstract}
Perfluorocarbon nanoparticles have been reported to deliver oxygen to tumors and reduce hypoxia-induced radioresistance, however few studies have been carried out to study its role in reducing hypoxia-induced chemoresistance. The oxygenation effect also varies dramatically between different perfluorocarbon formulations and protocols, and there have been no efficient tools to monitor dynamic changes of tumor oxygenation non-invasively. Our goal was to promote tumor oxygenation using perfluorooctyl bromide (PFOB) nanoemulsion and to assess its role in sensitizing tumors to cisplatin treatment. A novel optical imaging protocol was also created to monitor the dynamic changes of tumor oxygenation in real-time.

Methods: PFOB nanoemulsion with high oxygen-carrying capacity was prepared and administered to tumor-bearing mice intravenously. Tumor oxygenation was monitored using optical imaging with a hypoxia probe injected intratumorally, thus the oxygenation dynamics and best oxygenation protocol were determined. Various treatment groups were studied, and the tumor growth was monitored to evaluate the role of oxygenation in sensitizing tumors to cisplatin treatment.

Results: PFOB nanoemulsion with and without pre-oxygenation along with carbogen breathing resulted in much better tumor oxygenation compared to carbogen breathing alone, while PFOB with air breathing did not show significant increase in tumor oxygenation. Pre-oxygenated PFOB with carbogen breathing produced the most effective oxygenation as early as 5 min post administration. In vitro and in vivo data showed preoxygenated PFOB nanoemulsion with carbogen breathing could increase cisplatin-mediated apoptosis of cancer cells and inhibited tumor growth at a low dose of cisplatin $(1 \mathrm{mg} / \mathrm{kg})$ treatment. Furthermore, the treatment did not induce nephrotoxicity.

Conclusions: Preoxygenated PFOB nanoemulsion with carbogen breathing can effectively increase tumor oxygenation, which has a great potential to prevent/overcome hypoxia-induced chemotherapy resistance. In addition, optical imaging with intratumoral injection of the hypoxia probe was an efficient tool to monitor tumor oxygenation dynamics during PFOB administration, providing better understanding on oxygenation effects under different protocols.
\end{abstract}

Key words: perfluorooctyl bromide, hypoxia, oxygen delivery, cisplatin, chemoresistance.

\section{Introduction}

For decades, chemoresistance has been one of the greatest challenges to effective cancer therapy [1,2].
Clinical studies have shown that tumor hypoxia is one of the major factors associated with resistance to 
different types of cancer therapies [3]. Poor prognoses have been observed when using cisplatin (CPT) as a first-line chemotherapeutic agent in advanced stage cancer patients, and were reported to be largely due to hypoxia-induced drug resistance [4]. Hypoxia-induced resistance to cisplatin treatment has been associated with hypoxia-inducible factor 1 (HIF 1)-associated drug efflux, reduced apoptosis in hypoxic tumor, hypoxia-induced autophagy [5], and increased scavenging after conjugating with glutathione which is highly expressed in hypoxic tumor cells. Reducing tumor hypoxia or increasing tumor oxygenation has been recognized as an effective approach to reduce hypoxia-induced treatment resistance [6].

Many efforts have been made to improve tumor oxygenation during radiotherapy to overcome hypoxia-induced radio-resistance, including breathing hyperbaric oxygen, in situ production of oxygen from endogenous hydrogen peroxide $\left(\mathrm{H}_{2} \mathrm{O}_{2}\right)$, and tumor vasculature normalization with either drug molecules or enzymes to improve tumor blood flow [7-9]. However, the tumor oxygenation levels achieved by these methods are limited. Breathing hyperbaric oxygen is the only tumor oxygenation method currently used in the clinic in combination with radiation therapy, and success has been modest at best.

As part of the perfluorocarbon (PFC) family, perfluorooctyl bromide (PFOB) is a hydrophobic, chemically and biologically inert liquid that has been explored as a blood substitute for many years. PFOB has been used in patients for this purpose due to its high capacity of solubilizing oxygen and its biocompatibility [10]. This technology was then further extended to promote PFC-based tumor oxygenation to improve radiation and photodynamic therapy in pre-clinical animal models [11-15], but limited effort has been applied toward its use in chemotherapy. Tumor oxygenation using PFC-based agents may enhance the efficacy of chemotherapy, but more studies are needed to evaluate oxygenation effects on chemotherapy treatment. It has been recognized that even after decades of effort in tumor oxygenation, the clinical success is still limited with insufficient understanding of the oxygenation process. It is essential to develop techniques that can monitor hypoxia in solid tumors in real-time. Previous attempts have been made to measure tumor oxygen levels using needle-shaped polarographic electrode sensors [16-18]. However, this method is invasive, one-dimensional, and inefficient. It does not account for the heterogeneity of tumor oxygenation [19]. Tumor hypoxia may also be assessed using PET imaging with the hypoxia marker
${ }^{18}$ F-fluoromisonidazole (FMISO), but FMISO PET imaging takes at least two hours to get reasonable hypoxia contrast, and does not allow real-time hypoxia measurement [20,21]. MRI method using deoxyhemoglobin [22] in blood vessels could not provide accurate measurement on tissue oxygenation condition, as it is not indictive of the dissolved oxygen content. Pimonidazole immunostaining method $[11,12,15]$ requires tumor collection and cannot provide tumor oxygenation status before and after the treatment $[23,24]$. Therefore, we proposed to use an optical imaging method with intratumoral injection of a hypoxia probe to perform real-time monitoring of tumor oxygenation. Such method can greatly facilitate the development and evaluation of tumor oxygenation methods.

Considerable number of reports demonstrated that supplemental hyperoxic inhalation during PFC treatment would be crucial for effective oxygen delivery to tumors, whereas some recent studies reported that inhaling room air would be effective 24 $h$ after PFC administration [13,14,25]. In addition, oxygen-carrying capacity and circulating half-lives might be different for these formulations, thus the subsequent oxygenation efficiency may vary. Understanding the dynamics of tumor oxygenation in real-time for each PFC formulation is critical for establishing an optimal chemotherapeutic regimen.

Here, we present a novel strategy that aims at improving chemotherapeutic efficacy while minimizing the chemotherapy-induced toxicity based on a thorough understanding of the dynamics of tumor oxygenation using optical imaging. PFOB nanoemulsions were prepared as oxygen carriers. A variety of oxygenation methods were evaluated dynamically. Supplying supplementary hyperoxic breathing immediately after the administration of the PFOB nanoemulsion to tumor-bearing mice was necessary to maximize the oxygen retention in tumors. To promote an oxygenation burst effect, nanoemulsions were pre-oxygenated so that tumor oxygen levels could be promptly maximized. The combination of hyperoxic breathing with the administration of a pre-oxygenated PFOB nanoemulsion allowed for an immediate increase in tumor oxygenation, which was sustained for up to 6 hours. This approach allows for feasible co-administration of CPT and a pre-oxygenated PFOB nanoemulsion, ensuring adequate oxygenation levels in tumor tissues during the most critical period of the chemotherapeutic treatment. We successfully reduced the dose of CPT to a level that was low enough to avoid significant nephrotoxicity but still achieving inhibition of tumor growth in vivo. 


\section{Methods}

Detailed methodologies are provided as supplementary data.

\section{Preparation and characterization of PFOB nanoemulsion}

PFOB nanoemulsion was prepared using a heat-mediated emulsification method. Volume-based particle size distribution and zeta potential were determined via dynamic light scattering and anemometry laser doppler, respectively, on a Zetasizer Nano-ZS instrument (Malvern Instruments Ltd., UK). Transmission electron microscopy was used for characterizing particle morphology (Tecnai ${ }^{\mathrm{TM}}$ BioTWIN, FEI Company, Netherlands). Oxygenation was performed by bubbling pure $\mathrm{O}_{2}$ into glass vials containing the PFOB nanoemulsion for up to $3 \mathrm{~min}$. Oxygenation was monitored using the AL300 oxygen sensor probe attached to the NeoFox-GT fluorometer (Ocean Optics, USA) and using Image-iT ${ }^{\mathrm{TM}}$ hypoxia optical probe. In vitro oxygen release was simulated upon unsealing the air-tight vial for atmospheric re-equilibration. Supplementary Data Figure S1 depicts the apparatus used for $\mathrm{O}_{2}$ bubbling and in vitro oxygenation dynamics study.

\section{In vitro A549 cytotoxicity}

Hypoxic A549 cells were treated with 10-60 $\mu \mathrm{M}$ CPT followed by incubation for $24 \mathrm{~h}$ in ambient air $\left(21 \% \mathrm{O}_{2}\right)$, under carbogen (hyperoxic environment, $95 \% \mathrm{O}_{2}$ and $5 \% \mathrm{CO}_{2}$ ) for $3 \mathrm{~h}$ followed by $21 \mathrm{~h}$ incubation under ambient air, or with pre-oxygenated $0.4 \% \mathrm{v} / \mathrm{v}$ PFOB nanoemulsion in DMEM with incubation under carbogen as above. Normoxic cells were treated with CPT under equal conditions. Cell viability was assessed via CCK-8 method. Cells treated with saline and cultured under similar conditions were used as negative controls for each group.

\section{Animals and human lung cancer xenograft tumor model}

Severe combined immunodeficiency (SCID) mice were purchased from the Jackson Laboratory (Farmington, CT, USA) and housed in a pathogen-free animal facility at the University of Connecticut, School of Pharmacy. All animal experiments were conducted in accordance with regulations and guidelines of the Institutional Animal Care and Use Committee (A14-046). For the xenograft lung tumor model, A549 cells $\left(2 \times 10^{6}\right)$ suspended in $100 \mu \mathrm{L}$ of PBS buffer were subcutaneously injected into the right flanks of 6-8 week-old mice.

\section{In vivo optical imaging of real-time oxygenation levels in tumors}

Image-i $\mathrm{T}^{\mathrm{TM}}$ hypoxia reagent was intratumorally (50 $\mu \mathrm{L}, 1 \mathrm{mM}$ Image-iTTM) injected to tumors averaging $75 \mathrm{~mm}^{3}$, and the fluorescence signals monitored using the In Vivo Imaging System ${ }^{\circledR}$ (IVIS ${ }^{\circledR}$ ) Lumina LT Series III (excitation $=500 \mathrm{~nm}$, emission $=$ 650 nm, Perkin Elmer, Inc., USA). Animals were divided into four treatment groups $(n=5$ for each group): (1) OxySaline $+\mathrm{O}_{2}$ : pre-oxygenated saline breathing carbogen $\left(95 \% \mathrm{O}_{2}\right.$ and $\left.5 \% \quad \mathrm{CO}_{2}\right)$; (2) $\mathrm{OxyPN}+\mathrm{O}_{2}$ : pre-oxygenated $\mathrm{PFOB}$ nanoemulsion breathing carbogen; (3) $\mathrm{PN}+\mathrm{O}_{2}$ : non-oxygenated PFOB nanoemulsion breathing carbogen; and (4) OxyPN+Air: pre-oxygenated PFOB nanoemulsion breathing ambient air $\left(21 \% \mathrm{O}_{2}\right)$. Formulations or saline $(50 \mu \mathrm{l})$ was administered intravenously. Quantification was conducted by measuring the fluorescence intensity (average radiant efficiency) in the region of interest (ROI). Fluorescence intensity values were expressed as percentage of the initial fluorescence (prior to treatment) for each individual tumor.

To assess the impact of tumor oxygenation from circulating $\mathrm{PN}$ and $\mathrm{PN}$ accumulated in tumor tissue, mice were administered with OxyPN or saline and 24 $\mathrm{h}$ later the animals received an intratumoral dose of Image-i ${ }^{\mathrm{TM}}$ hypoxia reagent. Fluorescence signal was allowed to stabilize for $3 \mathrm{~h}$ and background signal was measured ( $27 \mathrm{~h}$ post initial treatment). Animals were allowed to breath carbogen $\left(95 \% \mathrm{O}_{2}\right.$ and $\left.5 \% \mathrm{CO}_{2}\right)$ for 1 $\mathrm{h}$ and re-imaged to assess the oxygenation effect attributed to lingering $\mathrm{PN}$ in blood circulation at 28 hours post injection.

\section{Ex vivo optical imaging of apoptosis in tumors}

Tumor apoptosis was evaluated in vivo by comparing three groups of tumor-bearing SCID mice $(\mathrm{n}=3)$ under different breathing regimens: (1) $5 \mathrm{mg} / \mathrm{kg}$ (i.v.) CPT breathing ambient air; (2) $5 \mathrm{mg} / \mathrm{kg}$ (i.v.) CPT breathing hyperoxic air (carbogen); and (3) 5 $\mathrm{mg} / \mathrm{kg}$ (i.v.) CPT plus pre-oxygenated $20 \%$ (v/v) PFOB nanoemulsion $(50 \mu \mathrm{L})$ breathing hyperoxic air. Breathing therapies were conducted for 3 hours post-injection. NIR-FLIVO ${ }^{\text {TM}}-747$ in vivo apoptosis assay agent was intravenously administered 48 hours post-treatment (50 $\mu \mathrm{L}$ i.v.; $1 x$ NIR-FLIVO ${ }^{\mathrm{TM}}$ solution per mouse). Four hours later, tumors were excised from mice and imaged using the IVIS ${ }^{\circledR}$ Lumina LT Series III (excitation $=747 \mathrm{~nm}$, emission $=776 \mathrm{~nm}$, Perkin Elmer, Inc., USA).

\section{In vivo antitumor efficacy $\&$ ex vivo apoptosis staining}

Tumor-bearing mice with average tumor sizes of 
60-75 $\mathrm{mm}^{3}(\mathrm{n}=4)$ were administered intravenously with $50 \mu \mathrm{L}$ of (1) saline; (2) CPT: free cisplatin (1 $\mathrm{mg} / \mathrm{kg})$; (3) $\mathrm{CPT}+\mathrm{O}_{2}$ : free cisplatin $(1 \mathrm{mg} / \mathrm{kg})$ under hyperoxic breathing for $3 \mathrm{~h}$; (4) OxyPN $+\mathrm{O}_{2}$ : pre-oxygenated PFOB nanoemulsion (PN) $(20 \% \mathrm{v} / \mathrm{v})$ under hyperoxic breathing for $3 \mathrm{~h}$; or (5) $\mathrm{CPT}+\mathrm{OxyPN}+\mathrm{O}_{2}$ : free cisplatin $(1 \mathrm{mg} / \mathrm{kg})$ and pre-oxygenated PFOB nanoemulsion $(20 \% \mathrm{v} / \mathrm{v})$ under hyperoxic breathing for $3 \mathrm{~h}$. Treatments were administered once per week for up to 40 days. The relative change in tumor volume was calculated using the $\mathrm{V} / \mathrm{V}_{0}$ ratio where $\mathrm{V}_{0}$ is the initial tumor volume.

At the end of the study, tumors were harvested, blocked in paraffin and sectioned into $5 \mu \mathrm{m}$-thick slices. Slices were stained with hematoxylin and eosin (H\&E) and terminal deoxynucleotidyl transferase (TdT) dUTP nick-end labeling (TUNEL). The number of apoptotic cells as a percentage of total cells in the HPF was determined ( $n=4,10 \mathrm{HPF} /$ tumor).

\section{Histopathological evaluation of toxicity}

At the end of the efficacy study, lungs, spleens, hearts, livers, and kidneys were harvested, blocked in paraffin and sectioned into $5 \mu \mathrm{m}$-thick slices, followed by H\&E staining. An additional tumor-bearing mouse group was treated with a high dose of CPT $(2 \mathrm{mg} / \mathrm{kg})$ as positive control, where toxicity is expected to occur.
Histopathological analysis was performed at the Connecticut Veterinary Medical Diagnostic Laboratory by a single-blinded Doctor of Veterinary Medicine.

\section{RESULTS}

\section{PFOB nanoemulsion characterization and oxygen-carrying capabilities}

The PFOB nanoemulsion comprised of spherical structures with a mean volume-based diameter of $197.4 \pm 12.3 \mathrm{~nm}$ (Figure 1A) and polydispersity index of $0.271 \pm 0.008$. Zeta potential was determined as $-19.1 \pm 3.9 \mathrm{mV}$ (Figure 1B), which is consistent with the polyethylene glycol coating of the particle's surface. Particle size distribution data was corroborated by the transmission electron microscopy images (Figure 1C), showing well-defined and monodisperse nanodroplets with sizes from 150 to 200 $\mathrm{nm}$. Particle shape and overall morphology is consistent with the intended formulation and characteristic of lipid-stabilized nanodroplets. PFOB's encapsulation efficiency was $80.8 \pm 7.3 \%$. PFOB loading was $134.7 \pm 12.1 \mu \mathrm{L}$ PFOB $/ \mathrm{mL}$, translating to percentage content of $16.2 \pm 1.5 \%(\mathrm{v} / \mathrm{v})$.
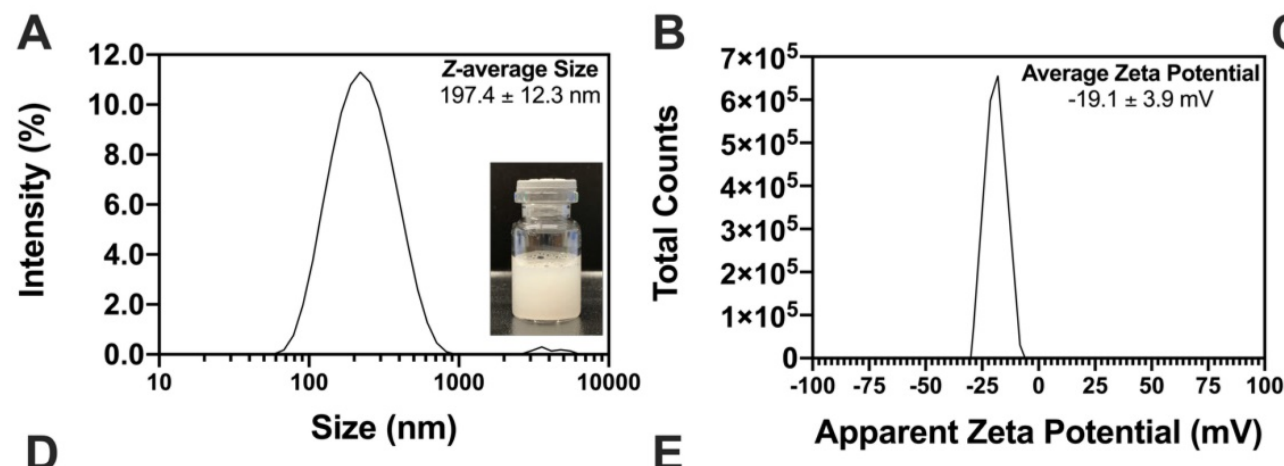

C

E

Apparent Zeta Potential (mV)
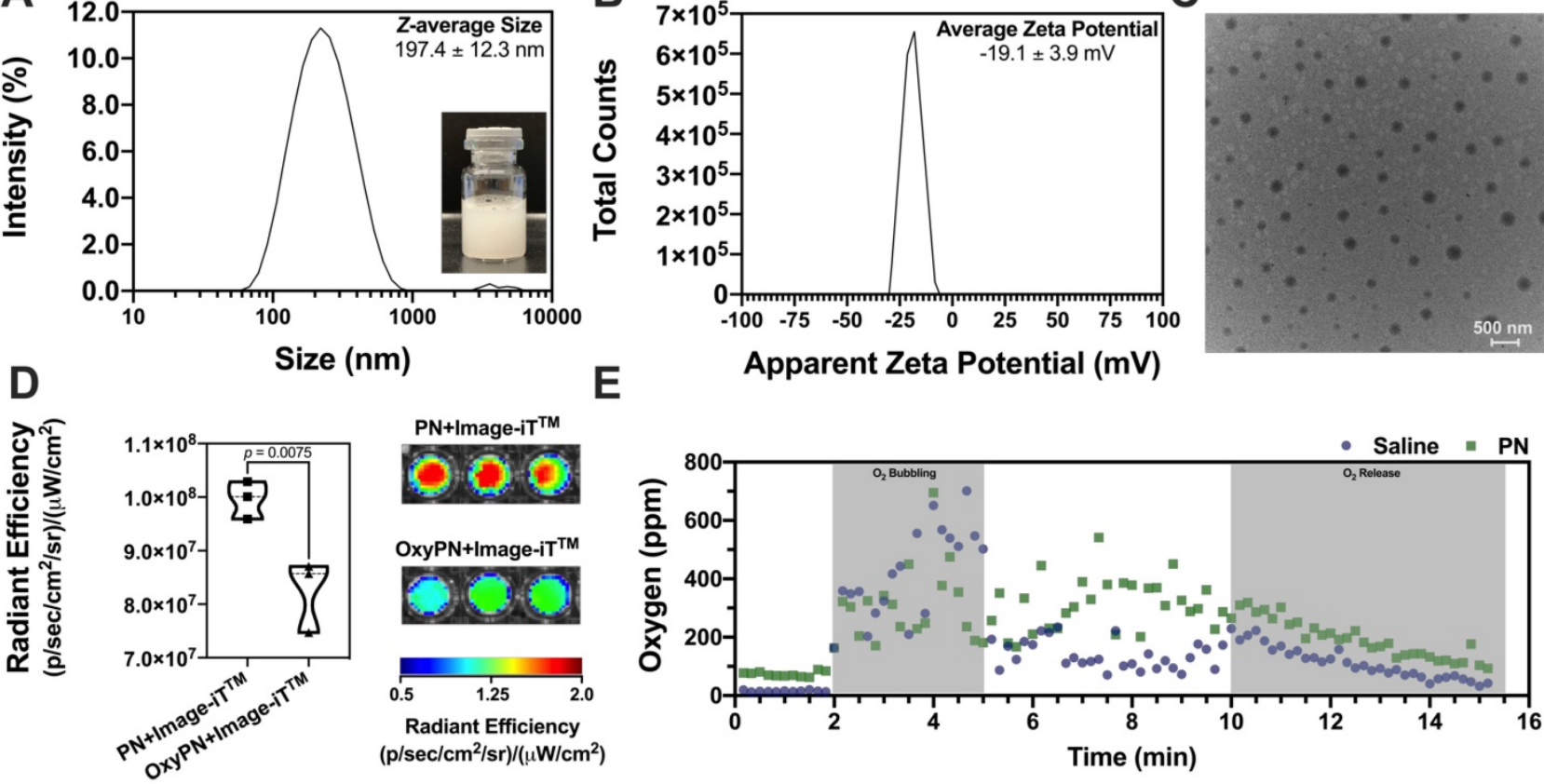

Figure 1. Characterization of PFOB nanoemulsion. (A) Volume-based particle size distribution of PFOB nanoemulsion. Insert shows a representative PFOB nanoemulsion preparation. (B) Apparent zeta potential distribution of PFOB nanoemulsion. (C) Representative transmission electron microscopy image of PFOB nanoemulsion. (D) In vitro impact of PFOB nanoemulsion oxygenation state on the radiant efficiency of the Image-iTTM hypoxia probe. (E) In vitro oxygenation dynamics of saline and PFOB nanoemulsion monitored via real-time fluorometry. $\mathrm{O}_{2}$ bubbling was conducted for 3 minutes for maximum oxygen saturation, followed by equilibration under hyperoxic atmosphere. $\mathrm{O}_{2}$ release was achieved by releasing the hyperoxic atmosphere and allowing equilibration with room air. 
The ability of PFOB nanoemulsions to carry oxygen was assessed. Upon oxygenation for 3 minutes with oxygen bubbling (Figure 1E), dissolved oxygen content in saline reached to $136.4 \pm 48.2 \mathrm{ppm}$, whereas PN reached to $325.2 \pm 86.4 \mathrm{ppm}$. Oxygen bubbling led to a supersaturated state with respect to the dissolved oxygen content (Figure 1E). PN showed enhanced capability of carrying oxygen when compared with saline, leading to a comparative 2.5 -fold increase in dissolved oxygen $(p<0.0001)$. The supersaturated oxygen levels are maintained under hyperoxic environment. Upon opening of the air-tight vial, the hyperoxic headspace quickly equilibrates with the room air $\left(\sim 21 \% \mathrm{O}_{2}\right)$ and promotes oxygen release from the supersaturated liquid, donating oxygen to the atmosphere following Henry's gas law. This behavior is clearly observed during the release portion of the oxygen dynamics monitoring data (Figure 1E), as further demonstrated in the supplementary data Figure S1.

In vitro Image-i $\mathrm{T}^{\mathrm{TM}}$ hypoxia probe response was evaluated as a function of the oxygenation level of the PFOB nanoemulsion (Figure 1D) before and after $\mathrm{O}_{2}$ bubbling, showcasing the dynamic reduction in fluorescence signal upon addition of OxyPN when compared with PN ( $p=0.0075)$. The dynamic response of the Image-iT ${ }^{\mathrm{TM}}$ probe, associated with the increased oxygen carrying capability of this formulation, showcases the potential of PN to deliver oxygen to tumors while allowing for real-time oxygenation level imaging.

\section{Pre-oxygenated PFOB nanoemulsion effectively improved CPT potency to A549 cells in vitro}

The susceptibility of A549 cells to cisplatin was assessed in vitro under hypoxic and normoxic conditions. Hypoxic A549 cells showed less susceptibility to cisplatin chemotherapy in vitro, as evidenced by the increased cell viability at equivalent CPT concentrations (Figure 2A). Normoxic A549 cells were 2-fold more susceptible to $\mathrm{CPT}$ at higher concentrations (30 and $60 \mu \mathrm{M}, p<0.0001$ ), showcasing the importance of maintaining adequate tumor cell oxygenation to enhance CPT cytotoxicity. The effect of OxyPN itself on the A549 cell viability was also assessed under both hyperoxic and normoxic conditions. It was found that OxyPN promotes negligible reduction of cell viability at the $0.4 \%(\mathrm{v} / \mathrm{v})$ concentration used for the in vitro studies $(p>0.05)$.

Using hypoxic A549 cells, the impact of OxyPN on the CPT cytotoxicity was investigated. $\mathrm{CPT}+\mathrm{O}_{2}$ and $\mathrm{CPT}+\mathrm{OxyPN}+\mathrm{O}_{2}$ groups showed increased cytotoxicity compared with groups treated with CPT alone under normoxic incubation (Figure 2B).
$\mathrm{CPT}+\mathrm{OxyPN}+\mathrm{O}_{2}$ group displayed a significant increase in cell death compared with the $\mathrm{CPT}+\mathrm{O}_{2}$ group, suggesting that hypoxic A549 cells were more sensitive to $\mathrm{CPT}$ under the $\mathrm{PFOB}$ nanoemulsion treatment.
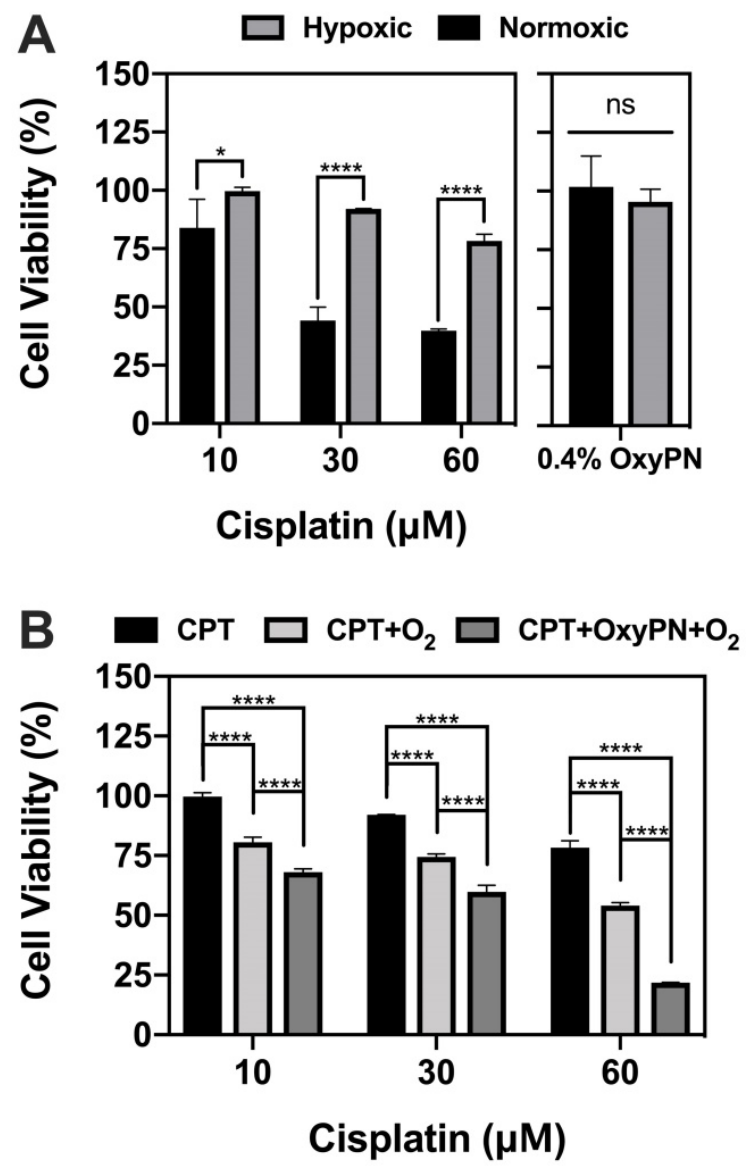

Figure 2. Viability of hypoxic and normoxic A549 cells 24 hours post-treatment. (A) Comparative viability of hypoxic and normoxic A549 cells upon treatment with only CPT or OxyPN. (B) Hypoxic cells treated with CPT, CPT under hyperoxic incubation $\left(\mathrm{CPT}+\mathrm{O}_{2}\right)$, and co-treatment of cisplatin and pre-oxygenated $\mathrm{PFOB}$ nanoemulsion under hyperoxic incubation $\left(\mathrm{CPT}+\mathrm{Oxy} \mathrm{PN}+\mathrm{O}_{2}\right)$ ( $n=3$; ns not significant, $* p<0.05, * * * * p<0.0001)$.

\section{In vivo real-time monitoring of tumor oxygenation levels during PFOB treatment}

Tumors exhibited a certain fluorescence signal due to their inherent hypoxic state upon Image-iT ${ }^{\mathrm{TM}}$ injection. The fluorescence signal became steady as early as $3 \mathrm{~h}$ post administration and remained reasonably steady for 12 to $24 \mathrm{~h}$. The total fluorescence intensity of each tumor at $12 \mathrm{~h}$ was set as baseline (timing of treatment administration) as the fluorescence readings were stable at this point, representing the hypoxic state prior to PFOB nanoemulsion treatment. Signals at other time points were normalized as percent change with respect to the baseline fluorescence. Figure $3 \mathrm{~A}$ showcases the fluorescence quantification for all time-points. 
Time-points in question refer to time elapsed since treatments (i.e. PN, OxyPN or saline) were administered. Figure 3B demonstrates selected time-points of interest. A full representative dataset is provided as supplementary data to this article (Figure 2S).

Significant signal reduction was observed $5 \mathrm{~min}$ post-injection of the pre-oxygenated PFOB nanoemulsion followed by hyperoxic breathing $\left(\mathrm{OxyPN}+\mathrm{O}_{2} ;\right.$ Figure 3). The rapid reduction in fluorescence signal indicated effective and rapid oxygen delivery to tumor tissues after injection. The normalized fluorescence intensity for $\mathrm{OxyPN}+\mathrm{O}_{2}$ and $\mathrm{PN}+\mathrm{O}_{2}$ groups were $62.8 \pm 4.1 \%$ and $83.5 \pm 12.8 \%$ respectively, which translates into a statistically-significant difference ( $p=0.0077$; Two-way ANOVA and Tukey's Multiple Comparison Test). Later time-points did not show differences between $\mathrm{OxyPN}+\mathrm{O}_{2}$ and $\mathrm{PN}+\mathrm{O}_{2}$ groups $(p>0.05)$. For the mice treated with the $\mathrm{PFOB}$ nanoemulsion without pre-oxygenation under hyperoxic breathing $\left(\mathrm{PN}+\mathrm{O}_{2}\right)$, the signal reduced gradually and reached a plateau in one hour. The signal started increasing approximately $4 \mathrm{~h}$ post treatment, although the animals were still under the hyperoxic breathing regimen.

At $28 \mathrm{~h}$ post-treatment, the effect of the remaining PFOB nanodroplets in circulation on the tumor oxygenation levels is minimal and indistinguishable from the saline group (Figure 3C). The normalized reduction in fluorescence intensity when comparing before and after $1 \mathrm{~h}$ hyperoxic breathing at $27 \mathrm{~h}$ post-treatment was similar between OxySaline and OxyPN groups (Figure 3D).

\section{Increase in caspase-mediated apoptosis via co-treatment with CPT and PFOB nanoemulsion}

Ex vivo apoptosis optical imaging assay was conducted to quantify cell apoptosis mediated via caspases signaling pathway. The NIR-FLIVO ${ }^{\text {TM}}-747$ reagent becomes fluorescent after binding to caspases. A stronger fluorescence intensity represents more caspases and thus increased cell apoptosis. As CPT induces cell apoptosis via the activation of caspases in A549 cells [26,27], this assay indicates how effective oxygenation treatment is in increasing CPT efficacy. As illustrated in Figure 4, more caspase-mediated apoptosis was observed in the group treated with $\mathrm{CPT}+\mathrm{OxyPN}+\mathrm{O}_{2} \quad(p=0.0046)$, implying that $\mathrm{PFOB}$ nanoemulsion-dependent enhancement in tumor oxygenation was responsible for a remarkable increase in caspase-mediated apoptosis.
A

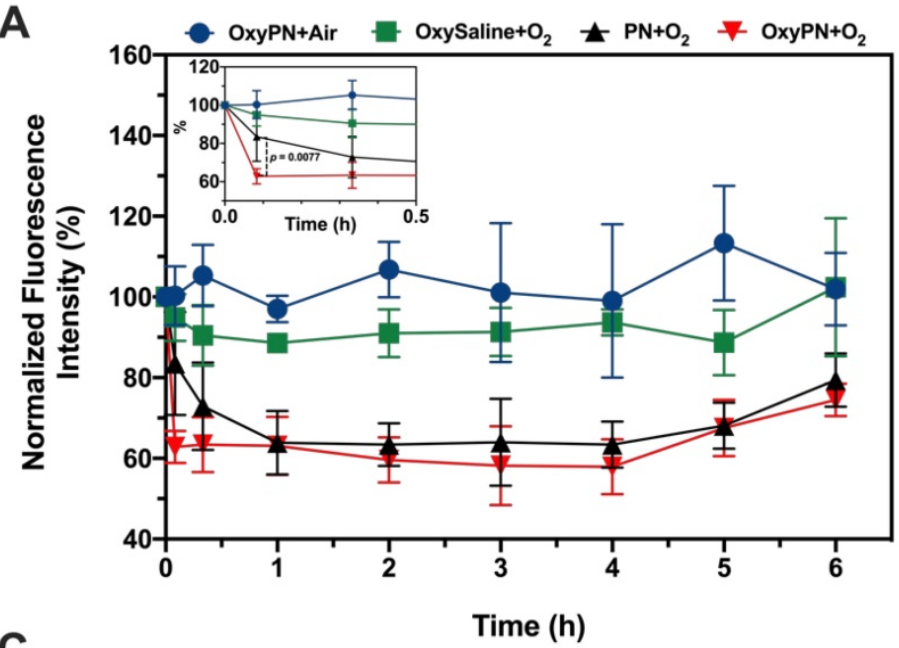

C
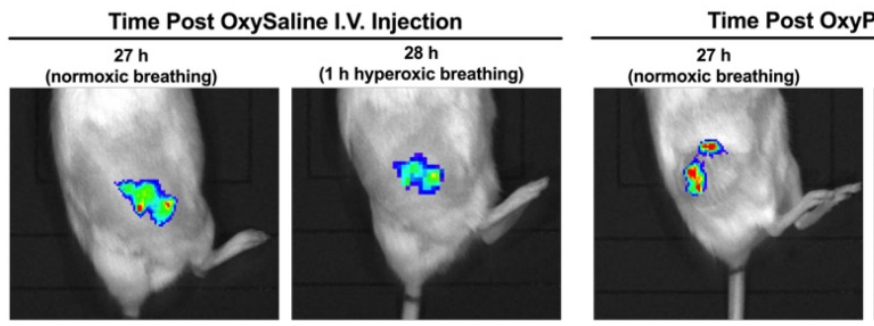

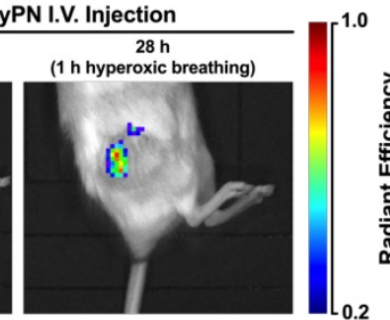

B

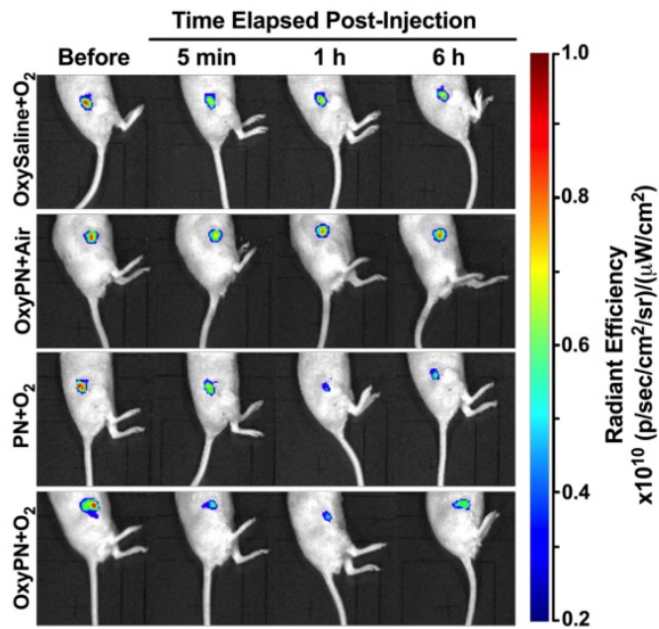

D

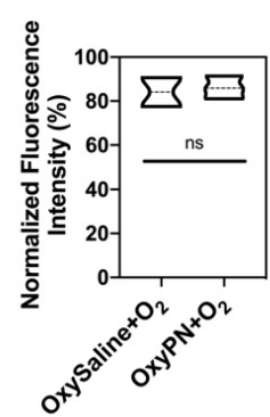

Figure 3. In vivo serial optical imaging of hypoxia in tumors. (A) Dynamic monitoring of hypoxic fluorescence from tumors ( $\mathrm{n}=3$ ). Higher level of fluorescence represents lower level of oxygenation in tumor. Insert highlights statistically-relevant differences at the first time-point. (B) Representative images of fluorescence from tumors at time zero- and at selected time-points. Full representative dataset is available as supplementary data (Figure S3). (C) Representative images of fluorescence from tumors 27 $\mathrm{h}$ post-treatment and $28 \mathrm{~h}$ post-treatment following $1 \mathrm{~h}$ hyperoxic breathing session. (D) Normalized radiant efficiency $28 \mathrm{~h}$ post-treatment as represented in (C). 


\section{Enhanced in vivo antitumor efficacy via co-treatment with CPT and PFOB nanoemulsion}

Based on the decrease in cancer cell viability observed in vitro (Figure 2) and the increase in caspase-mediated apoptosis in vivo (Figure 4), we postulate that increasing tumor oxygenation levels would result in more efficacious in vivo CPT efficacy. Studies involving simultaneous dosing of PFOB nanoemulsion and CPT were conducted since we observed that the pre-oxygenated PFOB nanoemulsion was capable of instantly delivering oxygen to the tumor tissues, with a long-lasting effect for up to $4 \mathrm{~h}$ (Figure 3A). Following CPT administration, we expected the drug to reach a maximum concentration in tumor tissues within the first hour [28]. Therefore, co-administering CPT and PFOB nanoemulsion at the same time would likely achieve synergistic efficacy.

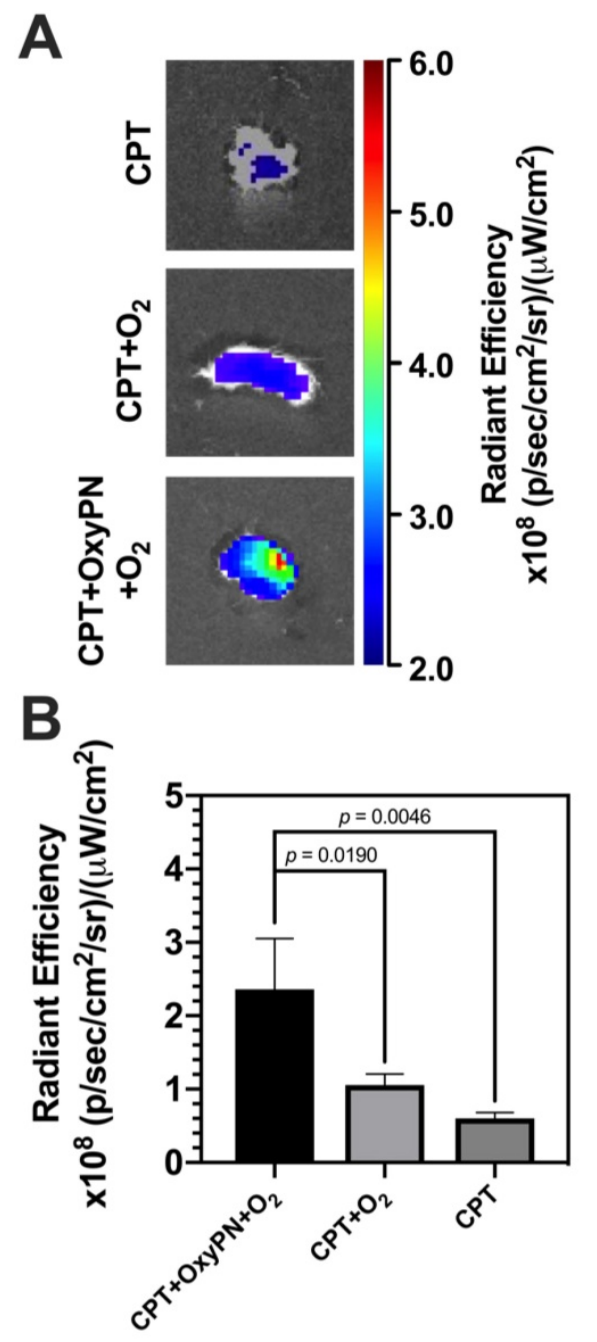

Figure 4. Ex vivo evaluation of caspase-mediated apoptosis in tumors. (A) Representative images of fluorescence from tumors harvested from mice 48 hours post-treatment. (B) Quantification of fluorescence signal from tumors $(n=3)$.
$\mathrm{CPT}+\mathrm{OxyPN}+\mathrm{O}_{2}$ group showed the most efficacious inhibitory effect on tumor growth (Figure $5 \mathrm{~A})$, consistent with in vitro observations. The relative tumor growth rate for the $\mathrm{CPT}+\mathrm{OxyPN}+\mathrm{O}_{2}$ group also reduced exponentially when compared with all other treatment groups $(p<0.0001$; Figure $5 \mathrm{~B})$. OxyPN $+\mathrm{O}_{2}$ group also displayed tumor growth inhibition compared with saline, even though no CPT was administered. Also, $\mathrm{CPT}+\mathrm{O}_{2}$ group displayed slightly enhanced tumor growth inhibition compared with CPT. These effects may be due to the known capacity of hyperoxic conditioning to inhibit tumor growth [29].

Analysis of high-power field (HPF) TUNEL-stained A549 tumors slices (Figure 5D) showed saline and $\mathrm{OxyPN}+\mathrm{O}_{2}$ did not produce a significant number of apoptotic cells per HPF. There was no difference between the saline group and $\mathrm{OxyPN}+\mathrm{O}_{2}$, even though $\mathrm{OxyPN}+\mathrm{O}_{2}$ treatment itself led to an inhibition in tumor growth in the in vivo efficacy study (Figure 5A).

All treatment groups that received $\mathrm{CPT}$ displayed a significant increase in apoptotic cells compared with saline $(p<0.0001)$. $\mathrm{CPT}+\mathrm{O}_{2}$ treatment increased the number of apoptotic cells compared with only CPT $(p<0.01)$. This is consistent with previous observations where hyperoxic breathing itself can slightly increase oxygen concentrations in tumor tissues. Corroborating previous findings, $\mathrm{CPT}+\mathrm{OxyPN}+\mathrm{O}_{2}$ group yielded a much greater number of apoptotic cells/HPF than the group treated only with $\mathrm{CPT}+\mathrm{O}_{2}(p<0.0001)$. In general, 2 - and 13-fold increase in apoptotic cells were found for $\mathrm{CPT}+\mathrm{OxyPN}+\mathrm{O}_{2}$ compared with $\mathrm{CPT}+\mathrm{O}_{2}$ and saline $(p<0.0001)$, respectively.

\section{Reduced CPT off-target toxicity following co-treatment with PFOB nanoemulsion}

During the treatment regimen, weight loss was observed in the $1 \mathrm{mg} / \mathrm{kg}$ CPT group and combinations containing $\mathrm{CPT}$, but not in the groups treated with saline or $\mathrm{OxyPN}+\mathrm{O}_{2}$ only (Supplementary Data, Figure S4). OxyPN itself did not change the natural growth rate of treated animals. Regarding kidney histopathological findings, saline-treated group and all groups treated with 1 $\mathrm{mg} / \mathrm{kg}$ cisplatin showed closed tubules, characteristic of regular renal function (Figure 6). No significant nephrotoxicity was observed in the co-treated groups. This allowed the use of a dose as low as $1 \mathrm{mg} / \mathrm{kg}$, while still maintaining efficacy due to the elevated oxygen levels in tumor tissues. For mice treated with 2 $\mathrm{mg} / \mathrm{kg} \mathrm{CPT}$, where toxicity is expected to occur, dilated tubules were present (Figure 6, yellow arrows) around the glomeruli (red arrows), suggesting 
accumulation of fluids in the tubule that could not be reabsorbed. No signs of oxygen-related toxicity (e.g. lung injury) were observed. No other signs of toxicity were observed in other organs as neither CPT nor the PFOB nanoemulsion caused histological damage to those tissues (Supplementary Data, Figure S5).

\section{Discussion}

We hypothesized that PFOB nanoemulsion-based oxygenation would promote better therapeutic efficacy by lowering hypoxia-induced chemoresistance. CPT is highly affected by hypoxia-induced chemoresistance [30], and it was the drug chosen for this study. A PFOB nanoemulsion (PN) formulation was developed and optimized to act as an oxygen carrier to promote tumor tissue oxygenation. The oxygen-carrying capability of PN was confirmed by successfully achieving 2-fold increase in dissolved oxygen content when compared with saline.

In vitro cell viability confirmed hypoxic A549 cells are less susceptible to CPT when compared with normoxic A549 cells, validating the merit of enhancing tumor oxygenation to promote better CPT efficacy. To test this hypothesis, in vitro cytotoxicity assay was performed to assess the impact of the PFOB nanoemulsion treatment on the cytotoxicity of CPT in hypoxic cells. Hypoxic cells were treated with various concentrations of CPT and incubated under $21 \%$ (normoxic) or $95 \% \mathrm{O}_{2}$ (hyperoxic), or with $\mathrm{CPT}$ plus $\mathrm{OxyPN}+\mathrm{O}_{2}$. The results indicate oxygenation of hypoxic A549 cells using OxyPN increased the cells' sensitivity to CPT. Since the OxyPN is able to carry much more oxygen compared with the cell culture medium, its presence allowed higher oxygenation levels to be achieved, increasing the cytotoxicity of cisplatin by overcoming hypoxia-induced chemoresistance in vitro. To bridge the translational gap between in vitro and in vivo efficacy, one must first understand the tumor oxygenation dynamics to properly tailor the treatment regimen (that is: the timing of administration of CPT and OxyPN).

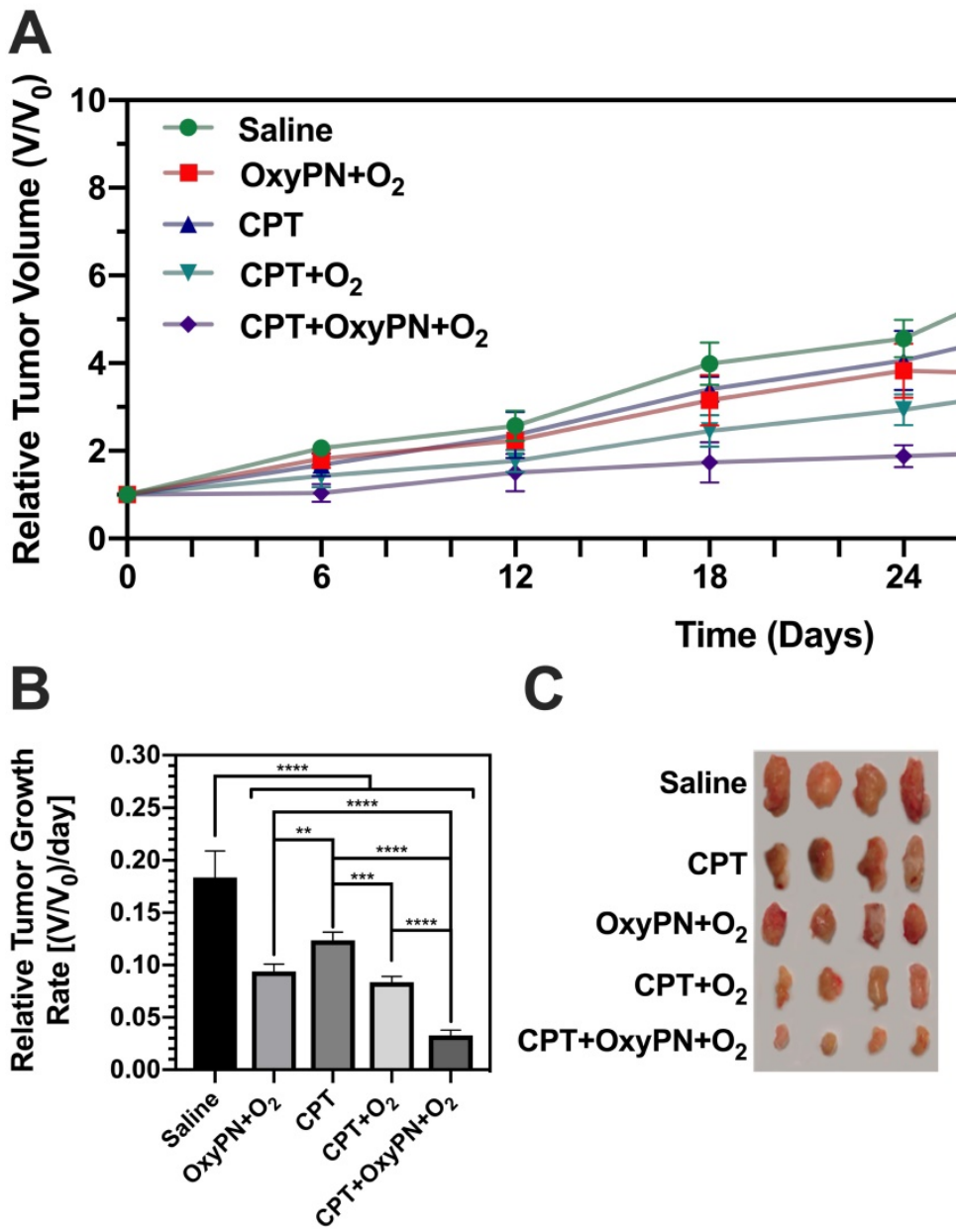

Figure 5. (A) Tumor growth of $A 549$ tumor-bearing mice treated weekly with saline, $\mathrm{OxyPN}+\mathrm{O}_{2}, \mathrm{CPT}, \mathrm{CPT}+\mathrm{O}_{2}$ or $\mathrm{CPT}+\mathrm{OxyPN}+\mathrm{O}_{2}(\mathrm{n}=4)$. (B) Relative tumor growth rate as specified in (A). (C) Tumors removed from each animal as specified in (A). (D) Percentage of apoptotic cells/HPF in tumors removed post efficacy study (TUNEL-stained slices; $\mathrm{n}=10) .{ }^{*} p<0.05, * * p<0.01, * * * p<0.001, * * * * p<0.0001$. 


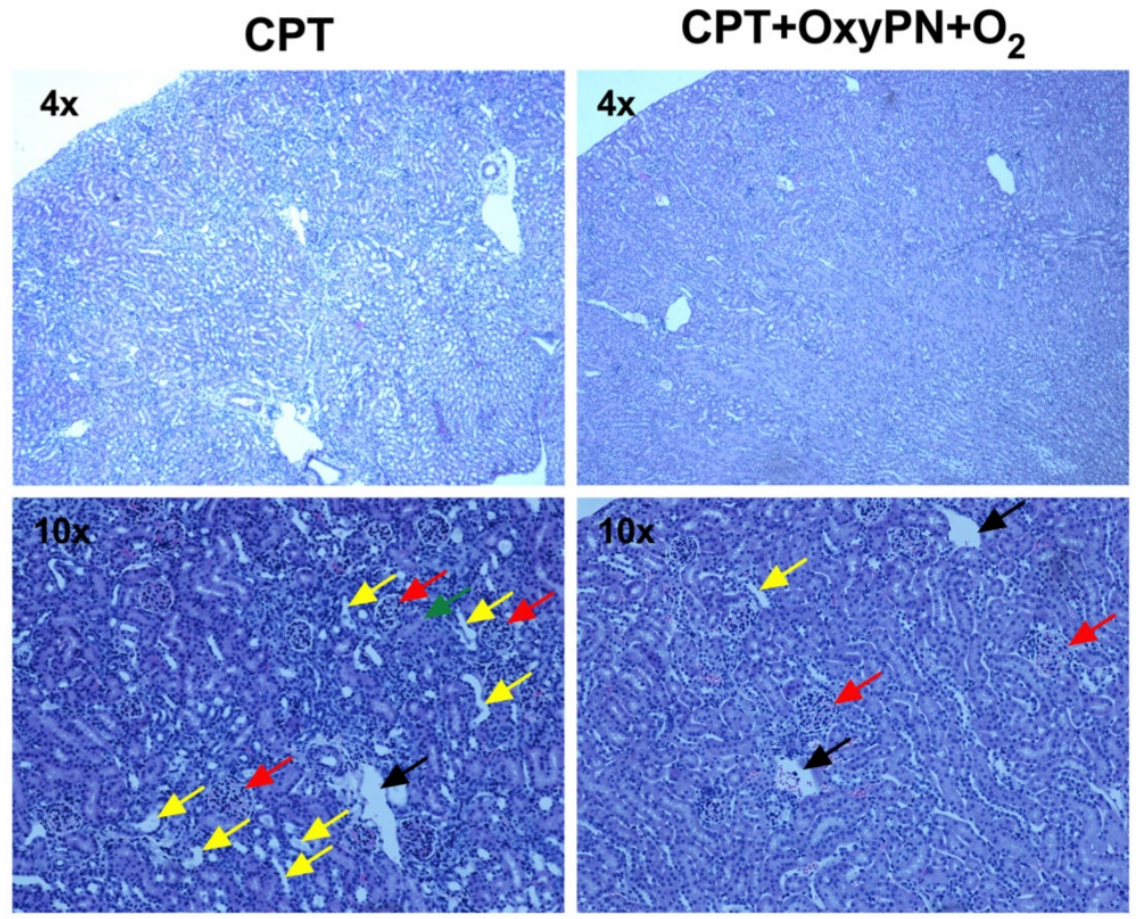

Figure 6. Representative images of H\&E staining of kidney slices. (Left) Representative micrographs from group treated with 2 mg/kg cisplatin (positive control). (Right) Representative micrographs of group treated with $1 \mathrm{mg} / \mathrm{kg}$ cisplatin and $\mathrm{CPT}+\mathrm{OxyPN}+\mathrm{O}_{2}$. Glomeruli, tubules, dilated tubules, and veins are marked by red, green, yellow and black arrows, respectively.

Illustrating the dynamic changes in tumor hypoxia following nanoparticle-mediated delivery of oxygen in real-time is of upmost importance at the pre-clinical stage, but still complicated due to inherent analytical and biological limitations. An optical imaging method for visualizing oxygen delivery to tumors was proposed in our work to better understand the oxygen delivery dynamics from OxyPN to tumor in vivo. Fluorescence signals from the Image-iT ${ }^{\mathrm{TM}}$ hypoxia probe decrease with increased oxygenation levels due to reaction quenching by oxygen and vice-versa. Since the quenching is reversible, it allows changes in oxygen levels to be instantly detected in real-time, promoting a much more efficient determination of tumor oxygenation dynamics during the OxyPN treatment. Considering nanoparticle accumulation in tumors does not occur instantaneously, the rapid delivery of oxygen observed after injection may not be related to nanoemulsion accumulation, but rather to the circulating nanoparticles that reach hypoxic areas with lower oxygen partial pressure, releasing oxygen. This pressure gradient would cause the oxygen dissolved in PFOB to diffuse out of nanoemulsion droplets into blood and further diffuse into adjacent tumor tissue. The dynamics of oxygen release from the PFOB nanoemulsion are similar to what is observed in vivo for red blood cells [31] and do not require nanoparticles to accumulate in the tumor tissues. Similarly, PFOB nanoemulsion in circulation would be able to "recharge" while passing through the pulmonary vasculature, which is oxygen-rich. The nanodroplets are able to carry dissolved oxygen and release it following Henry's gas law. The signal started to increase approximately $4 \mathrm{~h}$ post treatment, indicating decrease in tumor oxygenation, although the animals were still under the hyperoxic breathing regimen, demonstrating the most effective oxygen delivery period was within $4 \mathrm{~h}$ post-injection. From 4 -6 h, oxygenation still occurs but less efficiently. At 6 $h$, oxygenation is much less effective. This could be the result of clearance of the nanoemulsion droplets from circulation, reducing the number of particles available to effectively transport oxygen to the tumor's microvasculature. The half-life of the formulation in circulation was verified as $15.4 \mathrm{~h}$ using X-ray Computed Tomography (Supplementary Data, Figure S2) following an one-compartment intravenous bolus model [32]. Therefore, 4 hours post-injection, the remaining formulation in the circulation should correspond to roughly $80 \%$ of the total injected volume. This $20 \%$ loss in nanoemulsion concentration is apparently enough to reduce the oxygen partial pressure gradient effect driving the nanoparticle-mediated tumor oxygenation process.

It appears that effective tumor oxygenation by PN is highly dependent on supplemental hyperoxic breathing. Administration of OxyPN under air 
breathing (normoxic conditions) did not significantly change oxygenation levels in tumors, inferring the importance of supplementing oxygen breathing for a more effective tumor oxygenation via oxygen recharging of $\mathrm{PFOB}$ in the lungs. We postulate that circulating formulation can solubilize oxygen when these nanoparticles pass through the pulmonary vasculature, where the oxygen partial pressure is high due to the hyperoxic breathing regimen. This gradually improved oxygenation levels in tumor, as OxyPN circulates in blood, recharges with oxygen, and carries it onto hypoxic areas where oxygen will be delivered in the hypoxic microvasculature. It is noteworthy that this "oxygen recharging" process is one of the factors responsible for the high inter-individual variations commonly observed. Our result showed that pre-oxygenated $\mathrm{PN}$ (OxyPN) provided an almost instantaneous oxygenation effect, thus reducing inter-individual variations as the oxygenation process relies less on the pulmonary recharging compared with non-preoxygenated PFOB nanoemulsion.

The most effective tumor oxygenation was achieved with carbogen breathing within $4 \mathrm{~h}$ post OxyPN administration. As oxygen is delivered directly in the vasculature due to the oxygen partial pressure gradient, the maximum oxygenation period does not correlate with the time-frame of maximum nanoparticle accumulation in tumors (OxyPN tumor accumulation increased with time within $24 \mathrm{~h}$ post-injection; Supplementary Data Figure S6). The formulation remaining in blood circulation largely promoted oxygenation. These results support the importance of PN's oxygen-carrying capacity to promote improved oxygen transport through the vascular system and deliver to the hypoxic tumor tissues.

The impact of nanoemulsion concentration in circulation on its ability to increase tumor oxygenation was studied at 24 hours after receiving selected treatments (saline and $\mathrm{PN}$ ). The comparison among signals before and after hyperoxic breathing demonstrated no significant difference between OxySaline and OxyPN at such a late time-point. Therefore, it is confirmed tumor oxygenation is less effective longer than $6 \mathrm{~h}$ post administration of OxyPN due to nanoemulsion clearance from the body. Below such concentration, the increase in oxygen partial pressure in blood is not significant enough to drive tumor oxygenation further. Note this refers to dissolved oxygen levels in tumor, not tumor hypoxia state determined through biomarkers such as nitroimidazole. Image-iT ${ }^{\mathrm{TM}}$ probe measures the levels of free, dissolved, oxygen. Tumor accumulation of PN may contribute to the oxygenation but might not be substantial due to the limited amount of PN which eventually reaches tumors (in a scale of $1 \%$ of injected dose in tumors). Interestingly, a recent report has indicated little importance of carbogen breathing for tumor oxygenation [25]. In the referred report, hypoxia measurements were made $24 \mathrm{~h}$ post administration of PFOB droplets via PET imaging with $\left[{ }^{18} \mathrm{~F}\right] \mathrm{FAZA}$, unable to measure free oxygen levels. The referred study showed that hypoxia was reduced at 24 hours post injection of PFOB without carbogen breathing. For short term acute delivery of free oxygen, carbogen is recommended to promote an oxygen-rich environment for lung recharging of circulating PFOB nanodroplets, as demonstrated by the Image-iT ${ }^{\mathrm{TM}}$ tracking results which measures free oxygen. However, air breathing environment can potentially reduce hypoxia on a longer time-scale, as showcased by the referred study [25], which measured their results as a function of hypoxia state using $\left[{ }^{18} \mathrm{~F}\right] \mathrm{FAZA}$ PET. The reduction of tumor hypoxia at later time-points may not necessarily correlate to current oxygenation levels expressed as a function of free, dissolved, oxygen. Monitoring tumor oxygenation as a function of dissolved oxygen levels is relevant by a therapeutic perspective as a high free oxygen content in tumor is desired to promote effective chemo/radiotherapy [3,33].

Based on real-time oxygenation imaging, our findings confirm: (1) breathing room air does not improve the oxygenation in tumor tissues post PFOB nanoemulsion treatment as a function of free oxygen content; (2) PFOB nanoemulsion, with or without pre-oxygenation, is able to provide prolonged oxygen delivery under hyperoxic breathing; pre-oxygenated $\mathrm{PFOB}$ nanoemulsion provides a rapid oxygen supply to tumor tissues; (4) significant oxygenation occurs within a time-frame of $4 \mathrm{~h}$ post-injection of the PFOB nanoemulsion; (5) oxygen delivery likely occurs in the vasculature due to oxygen partial pressure differences, and does not require nanoparticle accumulation in solid tumor tissues.

With regards to the potential use of hypoxia probes in the clinic, Image-i $\mathrm{T}^{\mathrm{TM}}$ works within the NIR I region, thus limiting the depth and localization of the fluorescence source due to biological interference [34], and it is only used pre-clinically. However, a new generation of hypoxia probes are currently under development to operate within the NIR II region (1000 - $1700 \mathrm{~nm}$ ), drastically improving in vivo spatial resolution, signal-to-noise ratio and tissue penetration [35]. Also, current developments in clinical photoacoustic imaging may soon allow the use of such probes in the clinic [35]. In this sense, our findings lay solid ground for the exploration of novel 
hypoxia probes that operate under much lower biological interference as the reported experimental approach could be easily adapted.

CPT can promote a much greater tumor growth inhibition when treatment is performed under higher levels of oxygenation. Multiple elements have to be considered when discussing the role of oxygen in reducing the hypoxia-induced resistance to CPT. When oxygen is released from the PFOB nanoemulsion due to the lower oxygen partial pressure of the hypoxic tumor, it can foster the oxidation of thiol-containing molecules. Reduced numbers of thiol-containing molecules would aid in maximizing cellular uptake of CPT [36]. Also, oxygen should be sustainably supplied to prevent the recurrence of thiols due to the presence of active reductase. Besides the challenges of delivering oxygen in a timely manner, the pharmacokinetics associated with CPT distribution and accumulation in tumor tissues may also pose a challenge. It is known that intravenous injection of free CPT leads to peak concentrations in tumors shortly after administration, followed by a drug concentration reduction over time [37]. Maintaining high oxygen levels in tumors during CPT accumulation can be achieved by optimizing the dosing timing of both CPT and oxygen-carrying PFOB nanoemulsion. After the administration of OxyPN, tumor oxygen levels rapidly increased. To match this to CPT tumor accumulation, a simultaneous co-treatment of OxyPN and CPT can be applied to combine instantaneous oxygenation with the fast distribution of CPT in tumor tissues. This therapeutic approach was successfully validated in our pre-clinical model, where an exceptional tumor growth inhibition was achieved co-treating animals with CPT and OxyPN. In fact, the relative tumor growth rate for the $\mathrm{CPT}+\mathrm{OxyPN}+\mathrm{O}_{2}$ group showed a six-fold decrease when compared with the saline-treated control group.

Another advantage of $\mathrm{CPT}$ and OxyPN co-treatment is the possibility of reducing CPT doses while maintaining (or even increasing) $\mathrm{CPT}^{\prime}$ 's efficacy but reducing significantly possible toxic effects. Accumulated doses of CPT can induce irreversible kidney damage which is responsible for diminished excretion of erythropoietin and strongly limited regeneration of red blood cells [38,39]. In the presence of CPT-induced toxicity, anemia would cause the recurrence of hypoxia and the subsequent increase in tumor progression before the next cycle of treatment, thus limiting the overall therapeutic outcome. Therefore, achieving a therapeutic strategy that allows the use of lower doses of CPT while still promoting tumor growth inhibition is the main target of CPT-based chemotherapy. The inhibition of tumor growth may be achieved by developing strategies that aim at suppressing the population of therapy-resistant tumor cells, which are responsible for unmanageable tumor growth $[1,40,41]$. In particular, with respect to chemotherapy associated with PFOB nanoemulsion oxygenation, repeated high doses of chemo-drugs would increase the population of both drug-resistant and hypoxia-associated resistant cancer cells. Therefore, a low dose of CPT assisted with adequate PFOB nanoemulsion-based tumor oxygenation would lead to an improved inhibitory effect on tumor growth. To prove this hypothesis, we treated the subcutaneous tumor-bearing mice with $1 \mathrm{mg} / \mathrm{kg} \mathrm{CPT} \mathrm{together} \mathrm{with}$ the tailored PFOB nanoemulsion-based tumor oxygenation strategy. To our knowledge, $1 \mathrm{mg} / \mathrm{kg}$ CPT per week is the lowest dose that has ever been reported for significant inhibition of tumor growth. Under such conditions, we were able to achieve reasonable tumor growth inhibition without apparent signs of nephrotoxicity.

In summary, hypoxia-induced chemoresistance in cancer therapy creates a requirement for higher chemo-drug doses in order to achieve therapeutic success. This may lead to greater hypoxia, sustaining this negative cycle that makes cancer treatment even more difficult. We addressed this problem with a co-treatment strategy of low-dose of CPT $(1 \mathrm{mg} / \mathrm{kg})$ with adequate tumor oxygenation by a PFOB nanoemulsion formulation under hyperoxic breathing $\left(95 \% \mathrm{O}_{2}\right)$. A real-time in vivo hypoxia probe was used to demonstrate that PFOB nanoemulsion was able to sustain tumor oxygenation for up to $4 \mathrm{~h}$, transiently reducing tumor hypoxia. Pre-oxygenation of this nanoemulsion was necessary for fast oxygen delivery to tumor tissues. Due to the recharging capabilities of the PFOB nanoemulsion, hyperoxic breathing promoted continuous in vivo oxygen loading and consequently a more efficient tumor oxygenation. Co-administration of low dose CPT during this period of high tumor oxygenation allowed the successful inhibition of tumor growth compared with the administration of CPT alone. This chemotherapeutic strategy achieved effective therapy without significant nephrotoxicity.

\section{Conclusion}

PFOB nanoemulsion-based oxygenation has a great potential to prevent and/or overcome long-term hypoxia-induced chemotherapy resistance. Optical imaging allowed for dynamic measuring of oxygenation in tumors upon nanoemulsion administration in a pre-clinical murine model, providing novel understanding of the dynamics of nanoparticle-mediated tumor oxygenation and 
allowing for proper dosing design and timing to maximize cisplatin efficacy while reducing the dose administered. The use of optical imaging to understand the dynamics of tumor oxygenation upon nanoparticle administration is a novel approach, and it provides valuable knowledge for upcoming studies in the field of tumor oxygenation. Although due to the limited tissue penetration the current work targets imaging oxygenation in a preclinical study, with further developments in novel optical probes for hypoxia (i.e. NIR II probes), this approach may be adapted for use with such probes, narrowing the current translational gap between pre-clinical tumor oxygenation optical imaging - as reported in this study - and future clinical uses.

\section{Abbreviations}

18FMISO: 18F-fluoromisonidazole; CCK-8: Cell Counting Kit-8; CPT: cisplatin; DLS: Dynamic Light Scattering; DMEM: Dulbecco's Modified Eagle's Medium; DMSO: dimethyl sulfoxide; DSPE-PEG 2000:

1,2-distearoyl-sn-glycerol-3-phosphoethanolamine-N[amino(polyethylene glycol)-2000] ammonium salt; $\mathrm{H} \& \mathrm{E}$ : hematoxylin and eosin; $\mathrm{H}_{2} \mathrm{O}_{2}$ : hydrogen peroxide; HIF 1: hypoxia-inducible factor 1; HPF: high-power field; OxyPN: pre-oxygenated PFOB nanoemulsion; OxySaline: pre-oxygenated phosphate buffered saline; PBS: phosphate buffered saline; PET: positron emission tomography; PFC: perfluorocarbon; PFOB: perfluorooctyl bromide; PN: PFOB nanoemulsion; SCID: severe combined immunodeficiency mice; TEM: transmission electron microscopy; TUNEL: Terminal deoxynucleotidyl transferase dUTP nick-end labeling.

\section{Acknowledgements}

This work was partially supported by the University of Connecticut (X. Lu) and the National Institutes of Health (NIH) grant 5R21HL119975 (Z. Zhuang). The authors would like to express their appreciation to Dr. Laura Gonzalez-Fajardo for her assistance with the in vitro cell viability work.

\section{Supplementary Material}

Supplementary figures and tables.

http://www.ntno.org/v03p0223s1.pdf

\section{Competing Interests}

The authors have declared that no competing interest exists.

\section{References}

1. Enriquez-Navas PM, Kam Y, Das T, et al. Exploiting evolutionary principles to prolong tumor control in preclinical models of breast cancer. Sci Transl Med. 2016; 8: 327ra24.
2. Sosa Iglesias V, Giuranno L, Dubois LJ, et al. Drug Resistance in Non-Small Cell Lung Cancer: A Potential for NOTCH Targeting? Front Oncol. 2018; 8: 267.

3. Brown JM. Tumor Hypoxia in Cancer Therapy. In: Methods in Enzymology.Vol 435; 2007:295-321.

4. Wu HM, Jiang ZF, Ding PS, et al. Hypoxia-induced autophagy mediates cisplatin resistance in lung cancer cells. Sci Rep. 2015; 5: 1-15.

5. Rohwer N, Cramer T. Hypoxia-mediated drug resistance: Novel insights on the functional interaction of HIFs and cell death pathways. Drug Resist Updat. 2011; 14: 191-201.

6. Rockwell S, Dobrucki IT, Kim EY, et al. Hypoxia and radiation therapy: past history, ongoing research, and future promise. Curr Mol Med. 2009; 9: 442-58.

7. Gong H, Chao Y, Xiang J, et al. Hyaluronidase To Enhance Nanoparticle-Based Photodynamic Tumor Therapy. Nano Lett. 2016; 16: 2512-2521.

8. Yoon HY, Koo H, Choi KY, et al. Tumor-targeting hyaluronic acid nanoparticles for photodynamic imaging and therapy. Biomaterials. 2012; 33: 3980-3989.

9. Teicher BA, Holden SA, Ara G, et al. Restoration of Tumor Oxygenation After Cytotoxic Therapy by a Perflubron Emulsion/Carbogen Breathing. Cancer J Sci Am. 1995; 1: 43-48.

10. Follana R, Klein D, Krafft MP, et al. Prolonged shelf stability and biocompatibility of a concentrated injectable fluorocarbon emulsion. Biomater Artif Cells Immobilization Biotechnol. 1992; 20: 1059-61.

11. Song G, Liang C, Yi X, et al. Perfluorocarbon-Loaded Hollow $\mathrm{Bi}_{2} \mathrm{Se}_{3}$ Nanoparticles for Timely Supply of Oxygen under Near-Infrared Light to Enhance the Radiotherapy of Cancer. Adv Mater. 2016; 28: 2716-2723.

12. Song G, Ji C, Liang C, et al. TaOx decorated perfluorocarbon nanodroplets as oxygen reservoirs to overcome tumor hypoxia and enhance cancer radiotherapy. Biomaterials. 2017; 112: 257-263.

13. Xu L, Qiu X, Zhang Y, et al. Liposome encapsulated perfluorohexane enhances radiotherapy in mice without additional oxygen supply. J Transl Med. 2016; 14: 268.

14. Cheng $\mathrm{Y}$, Cheng $\mathrm{H}$, Jiang $\mathrm{C}$, et al. Perfluorocarbon nanoparticles enhance reactive oxygen levels and tumour growth inhibition in photodynamic therapy. Nat Commun. 2015; 6

15. Song X, Feng L, Liang C, et al. Ultrasound Triggered Tumor Oxygenation with Oxygen-Shuttle Nanoperfluorocarbon to Overcome Hypoxia-Associated Resistance in Cancer Therapies. Nano Lett. 2016; 16: 6145-6153.

16. Teicher BA, Holden SA, Dupuis NP, et al. Oxygenation of the Rat 91 Gliosarcoma and the Rat 13672 Mammary Carcinoma with Various Doses of a Hemoglobin Solution. Artif Cells, Blood Substitutes, Biotechnol. 1994; 22: 827-833.

17. Teicher BA, Schwartz GN, Dupuis NP, et al. Oxygenation of Human Tumor Xenografts in Nude Mice by a Perfluorochemical Emulsion and Carbogen Breathing. Artif Cells, Blood Substitutes, Biotechnol. 1994; 22: 1369-1375.

18. Teicher BA, Sotomayor EA, Dupuis NP, et al. Reduced oxygenation in a rat mammary carcinoma after chemo- or radiation therapy and reoxygenation with perflubron emulsion/carbogen breathing. J Cancer Res Clin Oncol. 1994; 120: 593-598.

19. Yeh KA, Biade S, Lanciano RM, et al. Polarographic needle electrode measurements of oxygen in rat prostate carcinomas: accuracy and reproducibility. Int J Radiat Oncol Biol Phys. 1995; 33: 111-8.

20. Bollineni VR, Kerner GSMA, Pruim J, et al. PET imaging of tumor hypoxia using 18F-fluoroazomycin arabinoside in stage III-IV non-small cell lung cancer patients. J Nucl Med. 2013; 54: 1175-80.

21. Lopci E, Grassi I, Chiti A, et al. PET radiopharmaceuticals for imaging of tumor hypoxia: a review of the evidence. Am J Nucl Med Mol Imaging. 2014; 4: 365-84.

22. Lee J, Hirano $\mathrm{Y}$, Fukunaga $\mathrm{M}$, et al. On the contribution of deoxy-hemoglobin to MRI gray-white matter phase contrast at high field. Neuroimage. 2010; 49: 193-8.

23. Nordsmark M, Loncaster J, Aquino-Parsons C, et al. Measurements of hypoxia using pimonidazole and polarographic oxygen-sensitive electrodes in human cervix carcinomas. Radiother Oncol. 2003; 67: 35-44.

24. Dhani NC, Serra S, Pintilie M, et al. Analysis of the intra- and intertumoral heterogeneity of hypoxia in pancreatic cancer patients receiving the nitroimidazole tracer pimonidazole. Br J Cancer. 2015; 113: 864-71.

25. Xiang Y, Bernards N, Hoang B, et al. Perfluorocarbon nanodroplets can reoxygenate hypoxic tumors in vivo without carbogen breathing. Nanotheranostics. 2019; 3: 135-144.

26. Grott M, Karakaya S, Mayer F, et al. Progesterone and estrogen prevent cisplatin-induced apoptosis of lung cancer cells. Anticancer Res. 2013; 33: 791-800.

27. Zhang X, Jiang S-J, Shang B, et al. Effects of histone deacetylase inhibitor trichostatin A combined with cisplatin on apoptosis of A549 cell line. Thorac Cancer 2015: 6: 202-208

28. Johnsson A, Olsson C, Nygren O, et al. Pharmacokinetics and tissue distribution of cisplatin in nude mice: platinum levels and cisplatin-DNA adducts. Cancer Chemother Pharmacol. 1995; 37: 23-31.

29. Raa A, Stansberg C, Steen VM, et al. Hyperoxia retards growth and induces apoptosis and loss of glands and blood vessels in DMBA-induced rat mammary tumors. BMC Cancer. 2007; 7: 23.

30. Wohlkoenig C, Leithner K, Deutsch A, et al. Hypoxia-induced cisplatin resistance is reversible and growth rate independent in lung cancer cells. Cancer Lett. 2011; 308: 134-43. 
31. Jensen FB. The dual roles of red blood cells in tissue oxygen delivery: oxygen carriers and regulators of local blood flow. J Exp Biol. 2009; 212: 3387-3393.

32. Zhang $\mathrm{Y}$, Huo $\mathrm{M}$, Zhou J, et al. PKSolver: An add-in program for pharmacokinetic and pharmacodynamic data analysis in Microsoft Excel. Comput Methods Programs Biomed. 2010; 99: 306-14.

33. Harrison L, Blackwell K. Hypoxia and Anemia: Factors in Decreased Sensitivity to Radiation Therapy and Chemotherapy? Oncologist. 2004; 9: 31-40.

34. Hong G, Antaris AL, Dai H. Near-infrared fluorophores for biomedical imaging. Nat Biomed Eng. 2017; 1: 0010.

35. Meng X, Zhang J, Sun Z, et al. Hypoxia-triggered single molecule probe for high-contrast NIR II/PA tumor imaging and robust photothermal therapy. Theranostics. 2018; 8: 6025-6034.

36. Zhu $\mathrm{H}$, Luo $\mathrm{H}$, Zhang $\mathrm{W}$, et al. Molecular mechanisms of cisplatin resistance in cervical cancer. Drug Des Devel Ther. 2016; 10: 1885-95.

37. Yu H, Tang Z, Zhang D, et al. Pharmacokinetics, biodistribution and in vivo efficacy of cisplatin loaded poly(l-glutamic acid)-g-methoxy poly(ethylene glycol) complex nanoparticles for tumor therapy. J Control Release. 2015; 205: 89-97.

38. Canaparo R, Casale F, Muntoni E, et al. Plasma erythropoietin concentrations in patients receiving intensive platinum or nonplatinum chemotherapy. $\mathrm{Br} \mathrm{J}$ Clin Pharmacol. 2000; 50: 146-53.

39. Miller RP, Tadagavadi RK, Ramesh G, Reeves WB. Mechanisms of Cisplatin nephrotoxicity. Toxins (Basel). 2010; 2: 2490-518.

40. Gatenby RA. A change of strategy in the war on cancer. Nature. 2009; 459: 508-509.

41. Gerlinger M, Swanton C. How Darwinian models inform therapeutic failure initiated by clonal heterogeneity in cancer medicine. Br J Cancer. 2010; 103: 1139-43. 